\title{
Interactions between dorsal and helix- loop-helix proteins initiate the differentiation of the embryonic mesoderm and neuroectoderm in Drosophila
}

\author{
Sergio González-Crespo and Michael Levine \\ Department of Biology, Center for Molecular Genetics, University of California at San Diego, \\ La Jolla, California 92093-0322 USA
}

\begin{abstract}
The dorsal ( $\mathrm{dl}$ ) morphogen has been implicated in the establishment of the embryonic mesoderm, neuroectoderm, and dorsal ectoderm in Drosophila. Here we show that the simultaneous reduction in the levels of $d l$ and any one of several helix-loop-helix (HLH) proteins results in severe disruptions in the formation of mesoderm and neuroectoderm. Certain triple heterozygous combinations essentially lack mesoderm as a result of a block in ventral furrow formation during gastrulation. HLH proteins that have been implicated previously in sex determination and neurogenesis (daughterless, achaete, and scute) are shown to be required for the formation of these embryonic tissues. Evidence is also presented that dl-HLH interactions involve the direct physical association of these proteins in solution mediated by the rel and HLH domains. We discuss the striking parallels in mesoderm formation and sex determination.
\end{abstract}

[Key Words: dorsal morphogen; helix-loop-helix proteins; Drosophila; embryonic mesoderm; neuroectoderm]

Received March 10, 1993; revised version accepted July 2, 1993.

In Drosophila, the specification of dorsoventral polarity is linked to the establishment of diverse embryonic tissues. The ventral-most cells of the embryo form mesoderm, those in ventrolateral regions differentiate into the neuroectoderm, and cells in the dorsal half of the embryo form dorsal ectoderm. A cascade of maternally expressed genes establish a nuclear gradient of dorsal (dl) protein, which functions as a morphogen by regulating gene expression in a concentration-dependent manner (Roth et al. 1989; Jiang and Levine 1993). $d l$ is a member of the rel family of transcription factors, which also includes the mammalian transcriptional activator NF- $\mathrm{B}$ and the chicken proto-oncogene c-rel (Steward 1987; Ip et al. 1991). Like the other members of the rel family, $d l$ is regulated at the level of nuclear transport. The protein is initially distributed throughout the cytoplasm of unfertilized eggs and early embryos. Approximately $90 \mathrm{~min}$ after fertilization, $d l$ protein in ventral and ventrolateral regions enters the nuclei, whereas protein in dorsal regions remains in the cytoplasm. This gradient in subcellular localization gives way to an absolute gradient, so that by the beginning of cellularization there are higher levels of $d l$ in ventral versus dorsal regions (Roth et al. 1989; Rushlow et al. 1989; Steward 1989).

Peak levels of $d l$ in ventral regions activate the mesoderm determinants twist (twi) (Thisse et al. 1988) and snail (sna) (Boulay et al. 1987). The promoters of both genes contain multiple, low-affinity $d l$-binding sites that directly mediate expression in the early embryo (Jiang et al. 1991; Pan et al. 1991; Thisse et al. 1991; Ip et al. 1992a). twi is a member of the helix-loop-helix (HLH) family of transcription factors (Murre et al. 1989) and is required for the activation of downstream mesodermal genes (Leptin 1991). sna is a zinc finger protein that acts as a repressor and blocks the expression of neuroectodermal regulatory genes in the presumptive mesoderm, thereby restricting them to lateral regions (Kosman et al. 1991; Leptin 1991; Rao et al. 1991).

Low levels of $d l$ in lateral regions activate the expression of rhomboid (rho), which encodes a putative transmembrane receptor required for the differentiation of a subset of the ventral epidermis that arises from the neuroectoderm (Bier et al. 1990). A 300-bp enhancer in the rho promoter is sufficient to direct lateral stripes of expression within the presumptive neuroectoderm. This enhancer contains high-affinity $d l$-binding sites and closely linked E-box sequences (HLH-binding sites) (Ip et al. 1992b). Mutations in either the $d l$ sites or E boxes result in catastrophic reductions in rho promoter activity, suggesting that the two classes of transcription factors function multiplicatively to activate $r$ ho expression (see below). The rho enhancer also contains sna-binding sites, which are responsible for repressing its expression in the presumptive mesoderm where there are high levels of $d l$ (Ip et al. 1992b). sna represses the expression of additional neuroectodermal regulatory genes, including 
lethal of scute (T3) (Campuzano et al. 1985; Cabrera et al. 1987, Romani et al. 1987). It also restricts the expression of single-minded (sim) (Crews et al. 1988; Thomas et al. 1988) and the m7 gene of the Enhancer of split complex [E(spl)] (Knust et al. 1987). These observations prompted the proposal that sna plays a key role in establishing the boundary between embryonic mesoderm and neuroectoderm (Kosman et al. 1991; Leptin 1991; Rao et al. 1991).

dl-HLH interactions are not only important for the regulation of rho in the neuroectoderm, but they also participate in the establishment of the mesoderm. For example, $d l /+$; twi / + double heterozygotes display variable disruptions in mesoderm differentiation, similar to those observed in $t w i^{-}$mutants (Simpson 1983). There is evidence that these disruptions arise from abnormal patterns of twi and sna expression (Kosman et al. 1991). A detailed molecular analysis revealed that twi binds to the sna promoter and functions multiplicatively with dl-binding sites to ensure strong, uniform expression of sna in the presumptive mesoderm (Ip et al. 1992a).

Here, we present evidence that $d l$ interacts with additional members of the HLH family, including the maternal products of daughterless (da) (Caudy et al. 1988b; Cronmiller et al. 1988) and the achaete-scute complex (AS-C). Dosage-sensitive interactions between $d l$, $d a$, AS-C, and twi are required for the specification of both the embryonic mesoderm and neuroectoderm. Various heterozygous combinations of these genes result in narrower limits of twi and sna expression. Reduced limits of sna cause ventral derepression of neuroectodermal regulatory genes normally restricted to lateral regions, including rho, T3, m7, and sim. Direct evidence that $d 1-$ $\mathrm{HLH}$ interactions are required for the activation of gene expression in the presumptive neuroectoderm was obtained by mutagenizing $\mathrm{E}$ boxes contained in the rho enhancer. Finally, evidence is presented that $d \mathrm{l}-\mathrm{HLH}$ interactions involve direct physical associations mediated by the rel and the HLH domains of these two distinct classes of transcription regulators.

\section{Results}

dl-da interactions are required for mesoderm formation

Dosage-sensitive interactions between $d l$ and twi were identified previously by analyzing $\mathrm{dl} / \mathrm{+}$; twi $/$ double heterozygotes (Simpson 1983; Kosman et al. 1991). These experiments involved mating $d l /+$ females with twi $/+$ males. Half of the embyros contained reduced levels of both proteins and showed severe disruptions in mesoderm formation. We decided to use this approach to determine whether $d l$ interacts with any other members of the HLH family. Particular efforts centered on HLH genes known to be expressed in early embryos, during the time when $d l$ initiates the dorsoventral pattern. $d a$ was tested first because it is maternally expressed and ubiquitously distributed in early embryos (Cronmiller and Cummings 1993).
All embryos derived from matings of $d l /+; d a /+$ females and normal males display abnormal patterns of twi and sna expression (Fig. 1), although the disruptions are somewhat variable from embryo to embryo. Figure 1, A-L, displays average distortions in the expression patterns. The expression limits of twi and sna are narrower than in wild type (Fig. 1, cf. E and $\mathrm{K}$ with $\mathrm{B}$ and $\mathrm{H}$ ). Normally, both genes are expressed in the ventral-most 18-20 cells, but in the double heterozygotes the limits include just 10-12 cells. In addition, there are gaps in expression near the cephalic furrow (Fig. $1 \mathrm{E}, \mathrm{J}, \mathrm{K})$, which persist during germ-band elongation (Fig. 1F,L). These gaps correlate with an expansion of the cephalic furrow, which is normally restricted to dorsolateral and lateral regions but extends into the presumptive mesoderm in double heterozygotes (Fig. 1F,L; data not shown). These alterations in the twi and sna patterns are similar to those observed in twi/ + embryos derived from $\mathrm{dl} /+$ females (Kosman et al. 1991).

Dosage-sensitive interactions between $d l$ and $d a$ depend solely on maternally expressed $d a$ products. Double heterozygotes obtained by mating $d l /+$ females and $d a /+$ males are viable and display completely normal twi and sna expression patterns (data not shown).

\section{dl interacts with $A S-C$ in mesoderm determination}

Certain HLH proteins encoded by the AS-C, such as T4, are also ubiquitously distributed in early embryos $/ \mathrm{Ca}$ brera et al. 1987; Romani et al. 1987). These proteins have been shown to interact genetically with $d a$ (Dambly-Chaudière et al. 1988) and form heterodimers that are potent transcriptional activators (Cabrera and Alonso 1991; Van Doren et al. 1991). For these reasons, we examined the possibility that $d l$ might interact with AS-C.

All embryos derived from matings of $D f(A S-C) /+$; $d l /+$ females and normal males show the same defects as those observed when maternal $d l$ and $d a$ products are reduced (Fig. 1). In particular, there is a narrowing in the twi and sna expression limits and a gap in the patterns near the presumptive cephalic furrow during cellularization that persists during germ band elongation (data not shown).

\section{Triple heterozygotes show a virtual loss of mesoderm}

To determine whether $d 1-\mathrm{HLH}$ interactions are redundant or cumulative, we analyzed the twi and sna expression patterns in triple heterozygotes. $d l /+; d a /+$ or $D f(A S-C) /+; d l /+$ females were mated with $t w i /+$ males. In these matings half of the resulting embryos contain reduced levels of $d l$ and two HLH proteins. Both classes of triple heterozygotes show similar severe disruptions in twi and sna expression (Fig. 2). During cellularization there are gaps in expression along the anteroposterior axis (Fig. 2A,D), and the limits are dramatically narrower, with expression spanning just the ventral-most 3-10 cells (Fig. 2B,E). The gap in expression near the cephalic furrow is greatly expanded as compared 

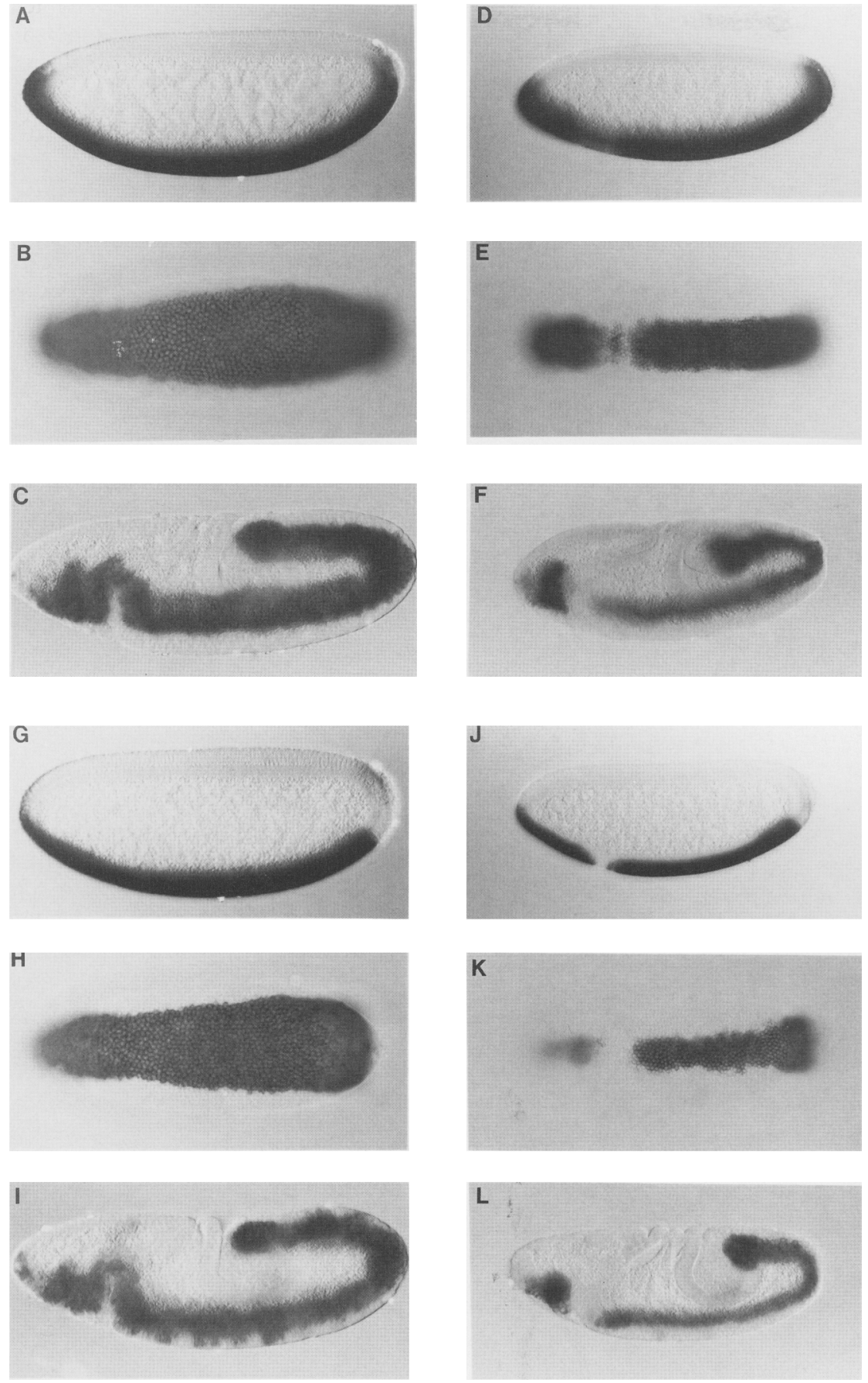
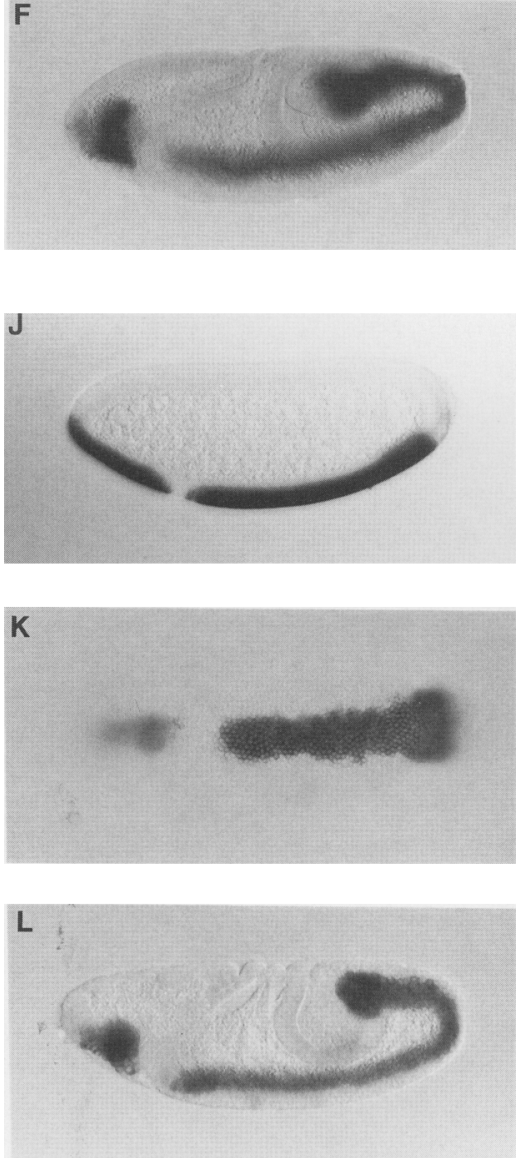

Figure 1. twi and sna expression in double heterozygotes for $d l$ and $d a$. Embryos were obtained by mating double-heterozygous females with Canton-S males. The embryos are oriented with anterior to the left. Expression patterns were visualized after in situ hybridization using digoxygenin-UTP-labeled RNA probes. $(A-C) t w i$ expression in wild-type (Canton-S) embryos. During cellularization, twi is expressed in a ventral band of $18-20$ cells $(B$, ventral view) that extends throughout the poles ( $A$, lateral view). This domain of expression represents the presumptive mesoderm and mesectoderm. twi expression is sustained in the mesoderm during germ band elongation $(C$, lateral view). $(D-F)$ $t w i$ expression in embryos derived from $d l^{8} ;+/+; d a^{I I B 31}$ females. At cellularization, the twi pattern is narrower than in wild type and spans only 12-14 cells $(E$, ventral view). In addition, there is a gap of expression in the vicinity of the presumptive cephalic furrow. Staining is detected in the invaginated mesoderm during germ band elongation $(F)$. Identical results were obtained with a different $d l$ mutation $\left(\mathrm{dl}^{6}\right.$; data not shown). (G-I) sna expression patterns in wild-type embryos. During cellularization the sna pattern spans the ventral-most 18 cells $(H$, ventral view) but is repressed at the poles $(G$, lateral view). There is transient expression of sna in the invaginated mesoderm $(I$, lateral view $)$. (JL) sna expression in embryos derived from $d l^{8} ;+/+; d a^{I I B 31}$ females. During cellularization, sna expression is restricted to a ventral band of just $8-10$ cells $(K$, ventral view). Moreover, there is a gap near the presumptive cephalic furrow $(J$, lateral view). This gap is sustained during germband elongation ( $L$, lateral view). Identical results were obtained in similar experiments using the null allele $d l^{6}$ (data not shown). with double heterozygotes (Fig. 2B and $\mathrm{E}_{\text {; }}$ cf. with Fig. 1E and $\mathrm{K})$. During germ band elongation there is nearly a complete loss of mesoderm invagination, such that internal twi and sna expression is restricted to posterior regions near the posterior midgut (PMG) (Fig. 2C,F). The ventral furrow fails to form in the triple heterozygotes (Fig. 3A,B), and the posterior patches of invaginated cells appear to arise through a process of ingression during germ band elongation (see Discussion).

These disruptions are not observed in embryos con- taining reduced doses of various HLH genes. For example, double heterozygotes for $d a$ and $t w i$ or AS-C and twi, as well as triple heterozygotes for $d a, \mathrm{AS}-\mathrm{C}$, and $t w i$ show completely normal patterns of $t w i$ and sna expression (data not shown).

\section{Neuroectoderm gene expression in $\mathrm{dl} / H L H$ heterozygotes}

Previous studies suggest that $d l-\mathrm{HLH}$ interactions are 
Figure 2. twi and sna expression in triple heterozygotes for $d l, d a, t w i$ and $d l$, AS-C, $t w i$. Double heterozygotes for $d l, d a$ or dl,AS-C females were mated with $t w i /+$ males, and the resulting embryos were hybridized with twi and sna RNA probes. $(A-$ C) twi expression in triple heterozygotes derived from a cross involving $D f(1) 260-1$ $(A S-C) /+; d l^{8} /+$ females and $t w i^{I D 96} /+$ males. By cellularization, the twi expression pattern includes a discontinuous band of just seven to eight cells. The most severe loss of expression occurs in the region of the presumptive cephalic furrow $(A$, lateral view; $B$, ventral view). These embryos lack a ventral furrow, and there is a near loss of invaginated mesoderm during germ band elongation $(C$, lateral view $)$. Identical results were obtained with the $d l^{6}$ and $s c^{10-1}$ alleles. In addition, a similar pattern was observed in $d l^{8}, d a^{I B 31}, t w i^{I D 96}$ triple heterozygotes (data not shown). $(D-F)$ sna expression in triple heterozygotes for $\mathrm{dl}^{8}$, $d a^{I B 31}$, and $t w i^{I D 96}$. By cellularization, sna expression is restricted to discontinuous patches of just three to eight cells in the ventral-most regions $(D$, lateral view; $E$, ventral view $)$. This pattern is sustained during germ-band elongation $(F$, lateral view $)$, although there is virtually no invaginated mesoderm. A similar defective sna pattern was observed in triple heterozygotes for $d l^{8}, D f(1) 260-1$ $(A S-C)$, and $t w i^{I D 96}$ (data not shown).

important for the regulation of neuroectodermal genes in lateral regions of cellularizing and gastrulating embryos (Ip et al. 1992b). We have investigated this issue further by examining the expression of various neuroectodermal regulatory genes in $d l / \mathrm{HLH}$ heterozygotes. Included in this study are three genes involved in neuronal differentiation; the $\mathrm{m} 7$ gene of the $\mathrm{E} / \mathrm{spl}$ ) complex, the T3 gene of AS-C, and $\operatorname{sim}$. In addition, rho expression was examined as it is one of the earliest markers for the neuroectoderm (Bier et al. 1990).

Each of these genes is expressed in ventrolateral regions that normally invaginate during gastrulation in embryos derived from $d l /+; d a /+$ or $D f(A S-C) /+; d l /+$ females (Fig. 4, cf. B, E, H, and $\mathrm{K}$ with $\mathrm{A}, \mathrm{D}, \mathrm{G}$, and J). Ventral derepression is particularly severe in the presumptive cephalic furrow where disruptions in the twi and sn $a$ patterns are most extreme (i.e., Fig. $1 \mathrm{E}, \mathrm{K}$ ). Triple heterozygotes [twi + embryos derived from either dl/ $+; d a /+$ or $D f(A S-C) /+; d l /+$ females] show severe ventral shifts of the $\operatorname{sim}, \mathrm{m} 7$, and $\mathrm{T} 3$ patterns, with expression extending into the ventral-most regions of the presumptive mesoderm (Fig. 4C, F, L). These shifts in expression correlate with the progressive narrowing of the sna pattern (i.e., Fig. 2E), which is probably responsible for excluding sim and $\mathrm{m} 7$ from the ventral-most regions. This correlation is particularly striking in triple heterozygotes, where the only ventral regions lacking rho and T3 coincide with residual patches of sna expression (Fig. 4I, L; see Fig. 2E).

dl-HLH interactions appear to be important for activating sim, $\mathrm{m} 7$, and $\mathrm{T} 3$ expression in lateral regions where there are low, limiting amounts of $d l$. Double and triple heterozygotes show a shift in expression, indicating that reduced levels of $d l$ and HLH proteins are unable to define the normal dorsal limits of expression. For example, T3 is normally expressed in a series of "hemistripes" that include an average of four to six cells (i.e., Fig. $4 \mathrm{~J}$ ). Simple derepression of T3 into the presumptive mesoderm should result in $\mathrm{T} 3$ stripes that encompass $\sim 28$ cells in ventral and ventrolateral regions. However, these stripes include only $20-22$ cells (Fig. 4L), indicating that the dorsal limits of the T3 pattern have shifted ventrally. Activation of rho does not appear to be sensitive to reductions in $d l$ and HLH products. The dorsal limits of the rho pattern are essentially normal in triple heterozygotes, so that the expanded pattern encompasses nearly the entire ventral half of the embryo $(\sim 36$ cells; Fig. 4I). This corresponds to the normal lateral stripes of rho expression in the neuroectoderm /each stripe is eight to nine cells in width) plus the presumptive mesoderm. It is conceivable that rho is buffered against changes in the levels of $d l$ and HLH proteins because its promoter contains numerous $d l$-binding sites that are closely linked to several classes of E-box sequences (see below).

\section{Role of $E$ boxes in neuroectoderm gene expression}

Additional evidence that $d \mathrm{l}-\mathrm{HLH}$ interactions are important for neuroectoderm gene expression was obtained by analyzing the expression of the rho NEE, a 600-bp enhancer located between -2.2 and $-1.6 \mathrm{~kb}$ upstream from the transcription start site. The NEE directs lateral 

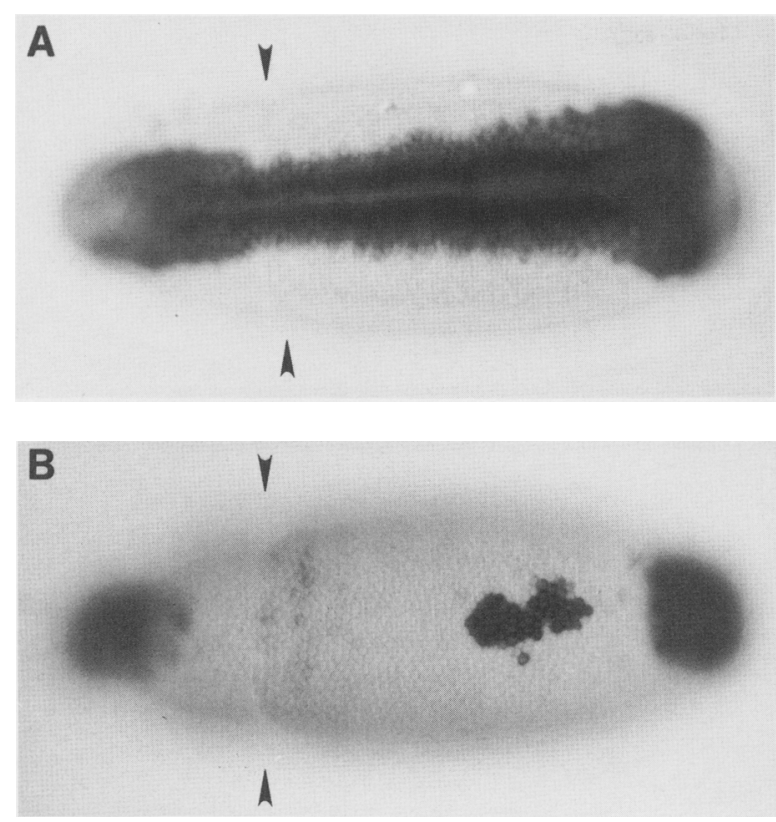

Figure 3. Triple heterozygotes lack a ventral furrow. (A) sna expression in a wild-type gastrulating embryo (ventral view). The 18 ventral-most cells invaginate through the ventral furrow. The cephalic furrow (arrowheads) does not extend through the presumptive mesoderm. (B) sna expression in a triple heterozygote for $d l^{8}, d a^{I I B 31}, t w i^{I D 96}$. This embryo is about the same age as the one shown in $A$. Staining is restricted to terminal regions and a patch in the presumptive abdomen. There is no ventral furrow, and the cephalic furrow (arrowheads) extends through the presumptive mesoderm. The same result was obtained in triple heterozygotes for $d l^{8}, D f(1) 260-1$ (AS-C), $t w i^{I D 96}$.

stripes of expression when attached to a lac $Z$ reporter gene (Fig. 5A; Ip et al. 1992b). It contains four dl-binding sites that mediate activation in ventral and ventrolateral regions and four sna-binding sites that are responsible for repression in ventral regions. In addition, the NEE contains several different $\mathrm{E}$ boxes.

The dorsal limits of NEE expression are essentially normal in $d l /+; d a /+$ and $D f(A S-C) /+; d l /+$ double heterozygotes (Fig. 5B). The slight ventral derepression of the pattern probably results from disruptions in the sna repressor. Perhaps the NEE is buffered against reductions in $d l$ and HLH expression because of functionally redundant $\mathrm{E}$ boxes.

To determine whether divergent HLH proteins might function redundantly to regulate the lateral expression of the NEE, we have mutagenized the E1, E2, E3, and E4 E boxes. Previous studies have shown that mutations in the E4 and E5 E boxes reduce, but do not abolish, NEE activity (Ip et al. 1992b). A mutagenized NEE lacking all four $\mathrm{E}$ boxes is completely inactive in embryos undergoing cellularization (data not shown). However, like the wild-type NEE, it is activated in the ventral-most regions of precellular embryos (Fig. 5C), prior to the time when the sna repressor is first detected. Evidence that $d l$ is sufficient for activation of the NEE in the presumptive mesoderm was obtained by analyzing the expression of the mutagenized NEE in sna mutants (Fig. 5D). Expression is restored in ventral, but not lateral, regions of cellularizing embryos; this corresponds to the time when the same enhancer is inactive in normal embryos. These results suggest that $d \mathrm{l}-\mathrm{HLH}$ interactions are required to direct expression in lateral regions where there are low levels of $d l$. High levels of $d l$ are sufficient for activation in ventral regions, but this is obscured in wild-type embryos by the sna repressor.

\section{The $\mathrm{dl}$ protein associates with HLH proteins in vitro}

To understand the molecular nature of $d l-H L H$ interactions, we determined whether the two classes of proteins can form heteromeric complexes. Radiolabeled fulllength $t w i, d a$, and $\mathrm{T} 4$ proteins were synthesized using a cell-free transcription-translation system (Fig. 6A) and incubated with an unlabeled, bacterially expressed glutathione $S$-transferase (GST)-dl fusion protein. The GST-dl protein contains the amino-terminal 378 amino acid residues of the $d l$ protein, which includes the entire rel domain. After immunoprecipitation with anti-dl antiserum, the immune complexes were analyzed by denaturing polyacrylamide gel electrophoresis. Labeled twi and $\mathrm{T} 4$, but not $d a$, were recovered by immunoprecipitation with anti- $d l$ antiserum only in the presence of the GST-dl fusion protein (Fig. 6B). The same results were obtained with two different anti- $d l$ antisera and in the presence of ethidium bromide to avoid nonspecific binding resulting from contaminating DNA (Lai and Herr 1992). These results suggest that $d l$ can form an activation complex with some, but not all, HLH proteins.

Because $d a$ is unable to interact in solution with $d l$, we decided to substitute its HLH domain with the one from $\mathrm{T} 4$, which can associate with $d l$. We constructed a chimeric $d a$ protein where the carboxy-terminal region (amino acids 553-710) containing the HLH domain was replaced by the corresponding carboxy-terminal region (amino acids 100-345) of T4. This chimeric da-T4 protein, containing the T4 HLH domain, now interacts with $d l$ in the coimmunoprecipitation assay (Fig. 6C). These results suggest that selective interactions between $d l$ and certain HLH proteins are mediated by the rel and HLH domains.

\section{Discussion}

The results presented in this study suggest that the establishment of the embryonic mesoderm and neuroectoderm depends on dosage-sensitive interactions between dl and a number of divergent HLH proteins. Some of these latter proteins have been implicated previously in sex determination and neurogenesis; this study provides the first evidence that HLH proteins such as $d a$ are also required for the specification of mesoderm. Triple heterozygotes containing reduced levels of $d l, d a$, and $t w i$ (or $d l$, AS-C, and twi) proteins essentially fail to form a ventral furrow and are almost completely devoid of in- 

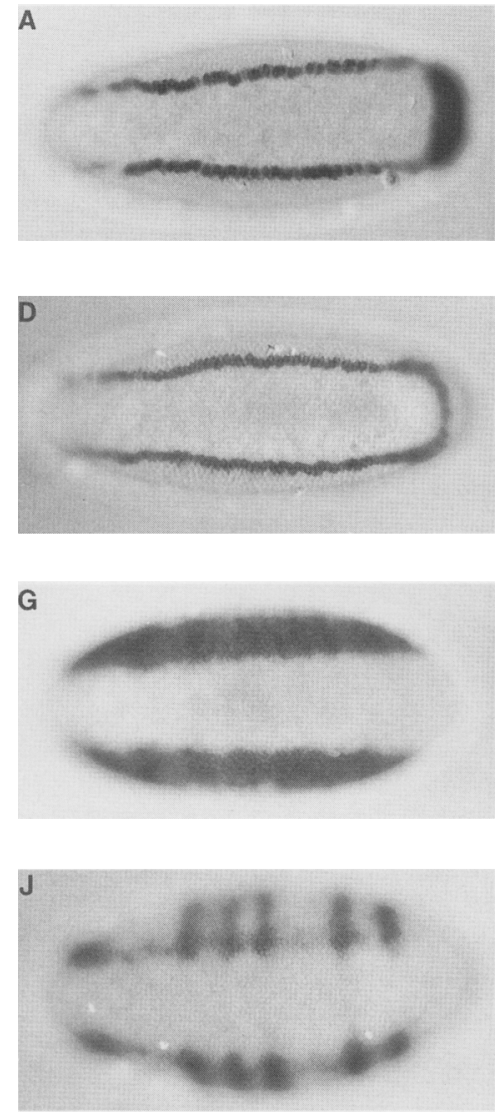
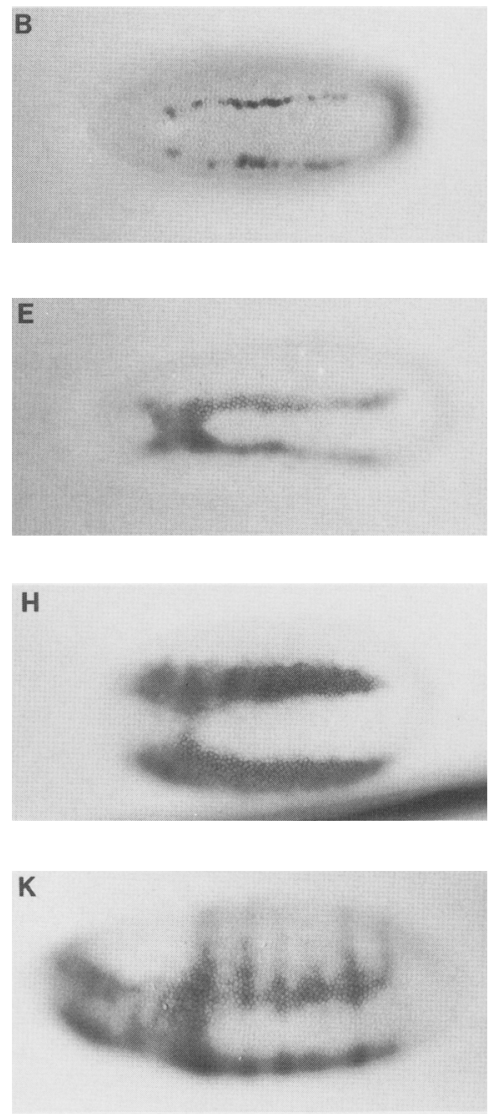
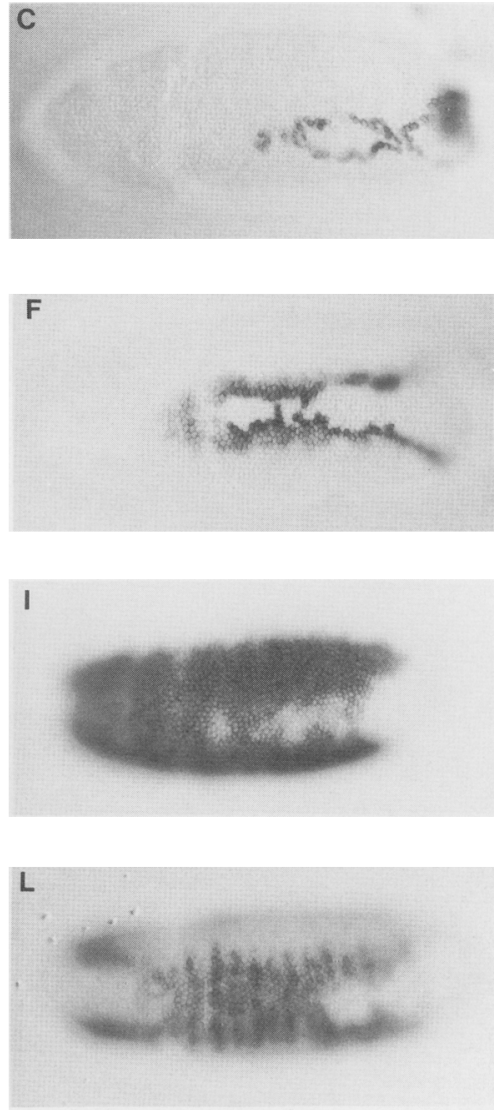

Figure 4. sim, $\mathrm{m} 7$, rho, and $\mathrm{T} 3$ expression in double and triple heterozygotes. $(A-C)$ sim expression. The wild-type pattern includes two one-cell-wide ventrolateral stripes along the $\mathrm{A}-\mathrm{P}$ axis $(A$, ventral view $)$. These stripes coincide with the presumptive mesectoderm and are separated by the presumptive mesoderm. The sim stripes shift ventrally and are closer together in double heterozygotes for $\mathrm{dl}^{8}$ and $d a^{I I B 31}(B$, ventral view). In addition, there are discontinuities in the stripes. sim expression is lost in anterior regions of triple heterozygotes for $d l^{8}, d a^{I I B 31}$, and $t w i^{I D 96}$, and the remaining stripes are discontinuous and shifted to ventral regions (C, ventral view). $(D-F) \mathrm{m} 7$ expression. After cellularization, the wild-type pattern includes two one-cell-wide ventrolateral stripes that are virtually identical to the sim pattern $(D$, ventral view). These stripes are shifted ventrally in $d l$ and $d a$ double heterozygotes (E, ventral view). In addition, the $\mathrm{m} 7$ pattern is joined in the vicinity of the presumptive cephalic furrow. The stripes include three to four cells in this embryo because it is younger (precellular) than the one in $D$. Initially, the $\mathrm{m} 7$ stripes are broad, but they are refined during cellularization. There is a severe ventral shift of the pattern in $d l, d a$, and $t w i$ triple heterozygotes ( $F$, ventral view). In addition, expression is lost in anterior regions. $(G-I)$ rho expression. The wild-type pattern includes two ventrolateral stripes that are $8-10$ cells wide and span most of the presumptive neuroectoderm $(G$, ventral view). These lateral stripes are expanded slightly toward more ventral regions in $d l$ and $d a$ double heterozygotes $(H$, ventral view). The expression pattern is nearly continuous in the region of the cephalic furrow. There is a severe ventral derepression of the pattern in $d l, d a$, and $t w i$ triple heterozygotes $(I$, ventrolateral view). $(J-L)$ T3 expression. The wild-type $\mathrm{T} 3$ pattern includes two discontinuous lateral stripes in the ventral half of the presumptive neuroectoderm $(J$, ventral view). In $d l$ and $d a$ double heterozygotes, these lateral stripes are shifted ventrally and the pattern is continuous in the vicinity of the cephalic furrow $(K$, ventrolateral view). In $d l, d a$, and $t w i$ triple heterozygotes there is a severe ventral derepression of the pattern $(L$, ventral view).

vaginated mesoderm. Biochemical studies suggest that the interactions between $d l$ and HLH proteins appear to be mediated by the conserved rel and HLH domains, respectively.

\section{The role of $\mathrm{dl}-\mathrm{HLH}$ interactions in development}

The sna expression pattern coincides with the presumptive mesoderm, and the sharp lateral borders of the pattern help to define the boundary between mesoderm and neuroectoderm (Boulay et al. 1987; Kosman et al. 1991;
Leptin 1991; Rao et al. 1991). It has been proposed that $d l-t w i$ interactions permit both high levels of $d l$ in ventral regions and intermediate levels of $d l$ in ventrolateral regions to activate sna to the same extent. According to this view, the shallow $d$ l gradient triggers a somewhat steeper $t w i$ gradient, and these function multiplicatively to define the sharp on/off borders of sna expression (Ip et al. 1992a).

Interactions between $d l, d a$, and AS-C are also important for specifying the presumptive mesoderm. The defective patterns of $t w i$ and sna expression observed in 


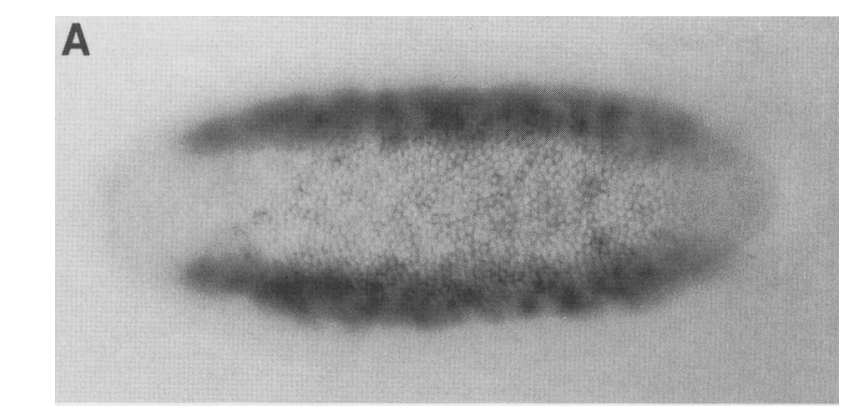

$100 \mathrm{bp}$

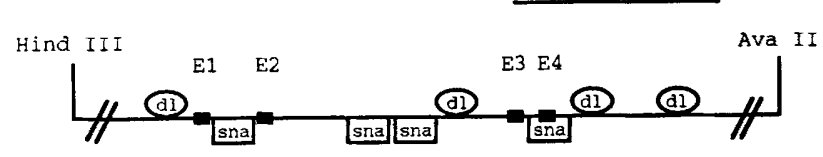

wild type
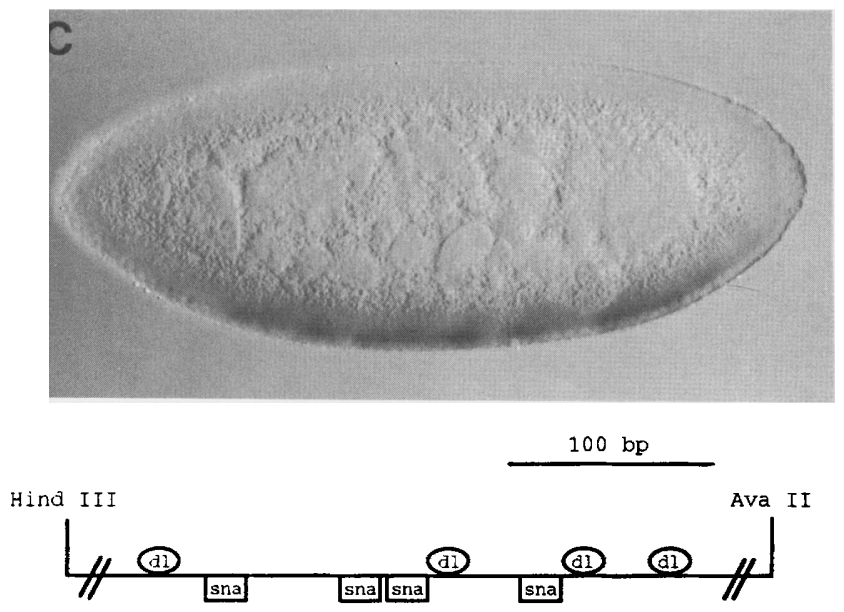

wild type

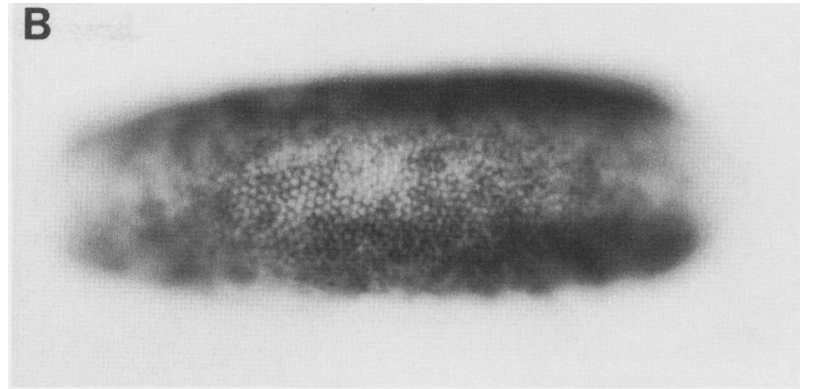

$100 \mathrm{bp}$
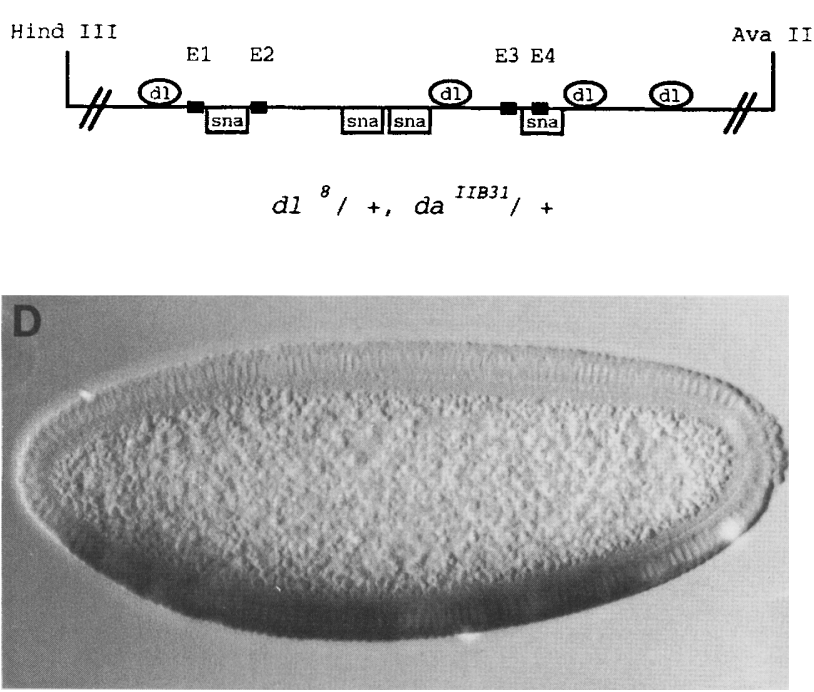

$100 \mathrm{bp}$

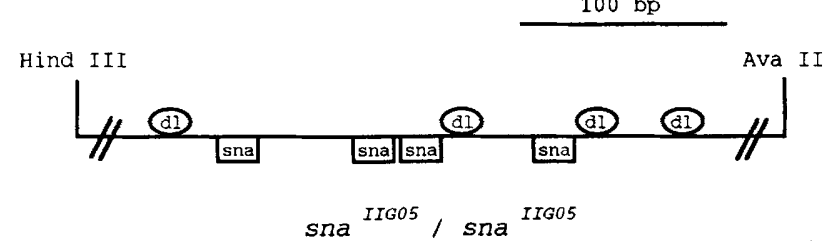

Figure 5. E boxes are required for neuroectoderm expression of the rho promoter. rho-lac $Z$ fusion genes were expressed in different genetic backgrounds. The expression patterns were visualized by in situ hybridization using a digoxygenin-UTP-labeled lacZ antisense RNA probe. The organization of the 600 -bp NEE is indicated below each embryo, including the location of relevant $E$ boxes as well as $d l$ - and sna-binding sites. $(A)$ The rho NEE pattern in a wild-type embryo (ventral view). Expression is restricted to lateral stripes in the presumptive neuroectoderm, similar to the endogenous pattern (cf. with Fig. $4 \mathrm{G}$ ). (B) NEE expression in a $d l^{8}$, $d a^{I I B 31}$ double heterozygote. There is a derepression of the pattern in ventral regions, similar to the endogenous rho pattern observed in this genetic background (cf. with $4 \mathrm{H}) .(C)$ Expression of a mutagenized NEE containing knockout mutations in the E1, E2, E3, and E4 E boxes. In this wild-type precellular embryo (lateral view) there is weak expression in ventral regions, similar to the initial pattern of the endogenous rho gene. However, during cellularization, expression is completely lost, indicating the importance of the $\mathrm{E}$ boxes for expression in the neuroectoderm. (D) Mutagenized NEE (same as $C$ ) in a cellularized sna embryo (lateral view). There is strong expression in the presumptive mesoderm, indicating that the $d l$-binding sites are sufficient to mediate expression in ventral regions. The same NEE is inactive in comparable wild-type embryos (data not shown).

double heterozygotes for $d l$ and either $d a$ or AS-C are similar to those seen in double heterozygotes for $d I, t w i$ (Fig. 1; Kosman et al. 1991). dl-da and dl-AS-C interactions may be distinct from the dl-twi interaction in that both $d a$ and AS-C products are homogeneously distributed throughout the early embryo. Thus, it would appear that the shallow $d l$ gradient can establish sharp patterns of target gene expression by interacting with either localized or ubiquitous HLH proteins. However, the pre- cise on/off borders of the sna pattern might rely on specific $d l-t w i$ interactions; reduced levels of $d a$ and/or T4 cause a narrowing of the twi pattern and perhaps a concomitant change in sna (see Fig. 1).

dl-HLH interactions are also important for the specification of the neuroectoderm. The dorsal limits of sim, $\mathrm{m} 7$, and T3 expression are shifted ventrally in double and triple heterozygotes (see Fig. 4). These results suggest that $d \mathrm{l}-\mathrm{HLH}$ interactions are required for the low 
A

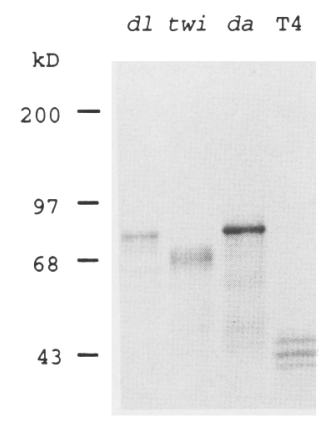

B

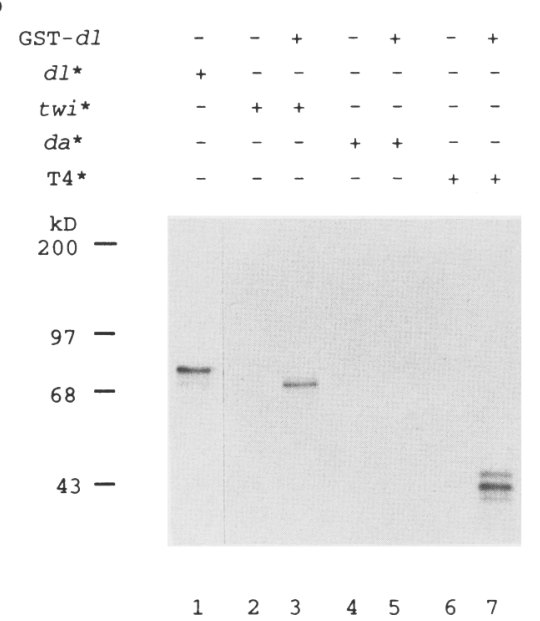

C

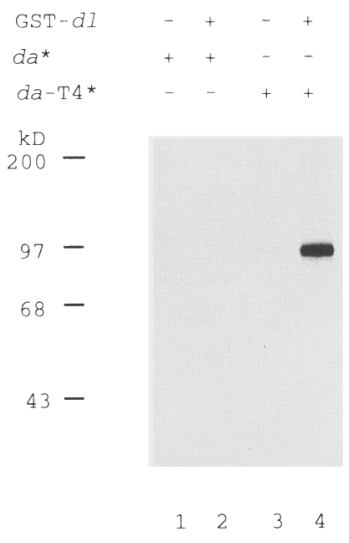

Figure 6. $d l$ forms complexes with $t w i$ and T4 but not $d a .(A)^{35} \mathrm{~S}$-Labeled $d l$, twi, $d a$, and T4 proteins synthesized in a reticulocyte extract. The proteins were fractionated on an SDS-polyacrylamide gel. Molecular mass markers are indicated at left. $(B)$ Immunoprecipitation assays. $d l$, twi, $d a$, and T4 proteins were incubated with an unlabeled GST-dl fusion protein and then immunoprecipitated with an anti-dl antiserum. Precipitated complexes were fractionated on an SDS-polyacrylamide gel and visualized by autoradiography. $(+)$ Proteins present in each mixture (top); $\left({ }^{*}\right)$ radiolabeled in vitro synthesized proteins. For example, lane 3 shows the immunoprecipitation complex obtained when the GST-dl fusion protein was incubated with labeled twi. Lane 2 shows the same experiment, except that the GST- $d l$ fusion protein was not added to the mixture. $|C|$ Immunoprecipitation assays using a chimeric $d a$ protein. A similar experiment as described in $B$ was performed using a chimeric $d a$ protein (referred to as $d a-\mathrm{T} 4$ ) where its carboxyterminal region containing the HLH domain was replaced by the carboxy-terminal region of T4 containing the T4 HLH domain.

levels of $d l$ in ventrolateral and lateral regions of the early embryo to activate target genes in the presumptive neuroectoderm. However, rho expression is relatively insensitive to dose, possibly owing to the close linkage and large number of $d l$ - and HLH-binding sites in the rho NEE.

A surprising finding of this study is that HLH proteins implicated previously in sex determination and neurogenesis are also essential for the determination of the mesoderm and neuroectoderm. $d a$ is a critical component of sex determination. Maternally encoded $d a$ products interact with both sis- $a$ /which encodes a leucine zipper protein) and sis- $b$ (the product of the T4 gene) to activate Sex lethal (Sxl) expression in precellular embryos (Cline 1989). In vitro binding assays suggest that da and T4 form heterodimers (Cabrera and Alonso 1991; Van Doren et al. 1991); perhaps these bind to the Sxl promoter and activate its transcription by interacting with sis- $a$ protein bound to neighboring sites. Like $d l-$ $\mathrm{HLH}$ interactions, the da/T4-sis- $a$ interaction is dosage sensitive. A twofold difference in the levels of sis- $a$ and $\mathrm{T} 4$ determines whether $S x l$ is on (females) or off (males). Similarly, a twofold reduction in the levels of $d l, d a, t w i$ or $d 1$, T4 (and/or T5), and twi result in a near on/off switch in sna expression (see Fig. 3). It is conceivable that the sis-a-da/T4 interaction is similar to $d l-\mathrm{HLH}$ interactions and involves direct protein associations (Fig. 6) and cooperative binding to DNA (Jiang and Levine 1993).

\section{Mechanism of $\mathrm{dl}-H L H$ interactions}

There are two types of models that could account for dosage-sensitive interactions between $d l$ and HLH proteins: cooperative binding to DNA or transcriptional synergism. According to the latter model, $d l$ and $\mathrm{HLH}$ proteins bind independently of one another but separately contact different rate-limiting components of the transcription complex. We favor the view that cooperative binding is the prime determinant of $d l-\mathrm{HLH}$ interactions because gel-retardation assays have demonstrated cooperative binding of $d l$ and $\mathrm{T} 4$ to neighboring sites on a short DNA fragment (Jiang and Levine 1993), and the present study indicates direct physical associations of the proteins.

The results of the in vitro binding assays (Fig. 6) suggest that $d l$ associates directly with a broad spectrum of HLH proteins. These complexes could bind to the promoters of target genes and activate transcription in a cooperative manner. For example, the sna promoter might be activated by a combination of $d l-t w i$ and $d l-\mathrm{T} 4$ complexes. However, a potential limitation of this model is that dosage-sensitive interactions are also observed for $d l$ and $d a$, yet these proteins fail to interact in coprecipitation assays. Additional DNA-binding assays offer a potential explanation for this observation (J. Jiang and M. Levine, unpubl.). A mixture of $d a$ and T4 proteins greatly increases cooperative binding of $d l$ to neighboring sites, although $d a$ alone has no effect on $d l$ binding. This increase in $d l$ cooperativity is the result of the enhanced binding activity of $\mathrm{T} 4$, which binds quite poorly as a homodimer but binds well as a heterodimer (Cabrera and Alonso 1991; Van Doren et al. 1991). Thus, it would appear that $d a$ interacts with $d l$ indirectly by facilitating the binding of HLH proteins (e.g., twi and T4) that can directly contact $d l$. 


\section{Evolutionary conservation of rel-HLH interactions}

The interactions that we have described for $d l$, a member of the rel family of transcription factors, and HLH proteins might be evolutionarily conserved. Coprecipitation of the $t w i$ and T4 proteins was obtained with a truncated dl protein that contains little more than the conserved rel domain. Moreover, twi and T4 were found to specifically associate with $\mathrm{dl}$, and both proteins are virtually unrelated except for the conserved HLH domain. These observations suggest that conserved sequences in $d l$ and HLH proteins are responsible for the interaction. If so, this interaction might apply to other systems involving members of the rel and HLH families such as the mammalian immunoglobulin $\mathrm{k}$-light-chain enhancer (Picard and Schaffner 1984) that contains closely linked NF-кBand HLH-binding sites.

\section{Materials and methods}

Fly stocks

The following fly stocks were used in this study. Wild type: Canton-S strain. $d l$ mutants: $d l^{8}$ and $d l^{6}$ are strong alleles that result in the loss of detectable protein (Roth et al. 1989). da: $d a^{I I B 31}$ is a null allele (Caudy et al. 1988a). AS-C: $D f(1) 260-1$ is a deficiency that spans the entire AS-C (Dambly-Chaudière et al. 1988). $s c^{10-1}$ disrupts the T4 and T5 genes in the AS-C (Villares and Cabrera 1987). $t w i$ : $t w i^{I D 96}$ causes the loss of detectable protein (Simpson 1983). sna: $s n a^{I I G O 5}$ is a strong allele (Simpson 1983).

All matings and embryo collections were performed at $25^{\circ} \mathrm{C}$.

\section{Whole-mount in situ hybridization}

Expression patterns were visualized in whole-mount preparations of embryos by in situ hybridization using antisense RNA probes labeled with digoxygenin-UTP and antidigoxygenin antibodies conjugated to alkaline phosphatase (Tautz and Pfeifle 1989; Jiang et al. 1991; Kosman et al. 1991). The stained embryos were photographed using Nomarski optics.

\section{Site-directed mutagenesis}

Point mutations were created in the E1, E2, E3, and E4 E boxes in the rho NEE using site-directed mutagenesis, as described previously (Ip et al. 1992a). The following mutagenic oligonucleotides were used: E1, AAGCTTCAGC /the normal sequence is AACATTTGGC); E2, GAGCAGAACG (the normal sequence is GACAAGTGCG); E3, CGCCAAGCTT (the normal sequence is CGCATATGTT); E4, ACGCGTTGTT (the normal sequence is AG $\overline{\mathrm{CA}}$ CATGTT).

The E-box consensus sequence is underlined. The mutations in the E 3 and E4 sites are the same as described by Ip et al. (1992b; formerly called $\mathrm{t} 1$ and $\mathrm{t} 2$ ).

\section{P-element transformation}

The mutagenized NEE was cloned into the P-element vector pWHL upstream of the hsp70 basal promoter (Driever et al. 1989). This plasmid (together with the $\Delta 2,3$ transposase helper plasmid) was injected in $w^{67}$ embryos. Three independent germline transformed strains were analyzed.

\section{Expression of bacterial GST-dl fusion protein}

Bacterial $d l$ protein was prepared as a fusion with GST using the pGEX KG expression plasmid and the Escherichia coli strain HB101 as described by Smith and Johnson (1988), Guan and Dixon (1991), and Ip et al. (1992a). The GST-dl fusion protein contains the amino-terminal 378-amino-acid residues of $d l$, which span the entire rel domain. The GST-dl protein that was used retains normal binding activity based on DNase I footprint assays (data not shown).

\section{In vitro transcription and translation}

In vitro-translated proteins were prepared from plasmids containing the entire $d l-, t w i-, d a$, and T4-coding sequences under the control of either the T7 (pAR) or SP6 (pBS) promoter. Linearized plasmids $(1 \mu \mathrm{g})$ were used as templates for in vitro transcription reactions, which were done at $37^{\circ} \mathrm{C}$ for $1 \mathrm{hr}$ with 50 units of T7 or SP6 RNA polymerase in $40 \mathrm{~mm}$ Tris- $\mathrm{HCl}$ (pH 7.5), $6 \mathrm{mM} \mathrm{MgCl}_{2}, 2 \mathrm{~mm}$ spermidine, $10 \mathrm{mM} \mathrm{NaCl}, 10 \mathrm{~mm}$ DTT, 0.1 $\mathrm{mg} / \mathrm{ml}$ of BSA, $0.5 \mathrm{~mm}$ each ATP, CTP, UTP, $0.05 \mathrm{mM} \mathrm{GTP}, 0.5$ $\mathrm{mM}$ GpppG, and 80 units of RNase inhibitor in a final volume of $50 \mu \mathrm{l}$. The reaction products were extracted with phenol/chloroform, ethanol precipitated, and analyzed by agarose gel electrophoresis. Typically, this procedure generated $\sim 1 \mu \mathrm{g}$ of RNA.

These RNAs (0.5 $\mu \mathrm{g}$ each) were translated at $30^{\circ} \mathrm{C}$ for $1 \mathrm{hr}$ in a rabbit reticulocyte lysate $(35 \mu \mathrm{l})$ in the presence of $0.02 \mathrm{~mm}$ amino acid mixture (minus methionine), $4 \mu$ l of $\left[{ }^{35} \mathrm{~S}\right.$ ]methionine $(1200 \mathrm{Ci} / \mathrm{mmole}, 10 \mathrm{mCi} / \mathrm{ml})$, and 40 units of RNase inhibitor in a final volume of $50 \mu$ l. The reaction products were analyzed after fractionation in SDS-polyacrylamide gels.

\section{Coimmunoprecipitation assays}

Aliquots of the GST-dl protein (100-300 ng) were mixed with $\sim 50,000 \mathrm{cpm}$ of ${ }^{35} \mathrm{~S}$-labeled protein prepared by in vitro translation. The mixtures were incubated at room temperature for 30 $\mathrm{min}$ in $50 \mu \mathrm{l}$ of NET-gel buffer $(50 \mathrm{mM}$ Tris- $\mathrm{HCl}$ at $\mathrm{pH} 7.5,150$ $\mathrm{mM} \mathrm{NaCl}, 0.1 \% \mathrm{NP}-40,1 \mathrm{~mm}$ EDTA at $\mathrm{pH} 8.0,0.25 \%$ gelatin); some of the experiments involved the addition of ethidium bromide, as described by Lai and Herr (1992). The mixture was then treated with protein A-agarose $(30 \mu 1)$ in a final volume of 230 $\mu l$ of NET-gel buffer at $4^{\circ} \mathrm{C}$ for $1 \mathrm{hr}$. The supernatant was incubated with a preabsorbed anti- $d l$ antiserum $(5 \mu l)$ at $4^{\circ} \mathrm{C}$ for 12 hr. Afterwards, protein A-agarose was added $(40 \mu l)$ and incubated at $4^{\circ} \mathrm{C}$ for $2 \mathrm{hr}$. After centrifugation, the pellet was washed twice with NET-gel buffer, twice with RIPA buffer $(50 \mathrm{~mm}$ Tris$\mathrm{HCl}$ at $\mathrm{pH} 7.5,150 \mathrm{~mm} \mathrm{NaCl}, 1 \% \mathrm{NP}-40,0.5 \%$ sodium deoxycholate, $0.1 \% \mathrm{SDS})$, and once in $10 \mathrm{~mm}$ Tris- $\mathrm{HCl}(\mathrm{pH} 7.5)$ and $0.1 \%$ NP-40. Each wash was performed with $1 \mathrm{ml}$ of buffer for $15 \mathrm{~min}$ at $4^{\circ} \mathrm{C}$. The final pellet was resuspended in $20 \mu \mathrm{l}$ of SDS gel-loading buffer $(50 \mathrm{~mm}$ Tris- $\mathrm{HCl}$ at $\mathrm{pH} 6.8,100 \mathrm{~mm} \mathrm{DTT}, 2 \%$ SDS, $0.1 \%$ bromphenol blue, and $10 \%$ glycerol), boiled for 3 min, and analyzed by autoradiography after fractionating on SDS-polyacrylamide gels.

\section{Acknowledgments}

We thank Tony Ip, David Kosman, Kazuaki Tatei, and Haini Cai for help and advice, Mark Van Doren and Andrew Singson for plasmids and suggestions, and James Posakony, Ethan Bier, and Cornellis Murre for encouragement. This work was funded by a grant from the National Institutes of Health (GM46638). S.G.C. is the recipient of a postdoctoral fellowship from the Spanish Ministerio de Educación y Ciencia.

The publication costs of this article were defrayed in part by 
payment of page charges. This article must therefore be hereby marked "advertisement" in accordance with 18 USC section 1734 solely to indicate this fact.

\section{References}

Bier, E., L.Y. Jan, and Y.-N. Jan. 1990. rhomboid, a gene required for dorsoventral axis establishment and peripheral nervous system development in Drosophila melanogaster. Genes \& Dev. 4: 190-203.

Boulay, J.L., C. Dennefeld, and A. Alberga. 1987. The Drosophila developmental gene snail encodes a protein with nucleic acid binding fingers. Nature 330: 395-398.

Cabrera, C.V. and M.C. Alonso. 1991. Transcriptional activation by heterodimers of the achaete-scute and daughterless gene products of Drosophila. EMBO J. 10:(10) 2965-2973.

Cabrera, C.V., A. Martínez-Arias, and M. Bate. 1987. The expression of three members of the achaete-scute complex correlates with neuroblast segregation in Drosophila. Cell 50: 425-433.

Campuzano, S., L. Carramolino, C.V. Cabrera, M. Ruiz-Gómez, R. Villares, A. Boronat, and J. Modolell. 1985. Molecular genetics of the achaete-scute gene complex of D. melanogaster. Cell 40: 327-338.

Caudy, M., E.H. Grell, C. Dambly-Chaudière, A. Ghysen, L.Y. Jan, and Y.N. Jan. 1988a. The maternal sex determination gene daughterless has zygotic activity necessary for the formation of peripheral neurons in Drosophila. Genes \& Dev. 2: 843-852.

Caudy, M., H. Vaessin, M. Brand, R. Tuma, L.Y. Jan, and Y.N. Jan. 1988b. daughterless, a Drosophila gene essential for both neurogenesis and sex determination, has sequence similarities to myc and the achaete-scute complex. Cell 55: 1061-1067.

Cline, T.W. 1989. The affairs of daughterless and the promiscuity of developmental regulators. Cell 59: 231-234.

Crews, S.T., J.B. Thomas, and C.S. Goodman. 1988. The Drosophila single-minded gene encodes a nuclear protein with sequence similarity to the per gene product. Cell 52: 143151.

Cronmiller, C. and C.A. Cummings. 1993. The daughterless gene product in Drosophila is a nuclear protein that is broadly expressed throughout the organism during development. Mech. Dev. (in press).

Cronmiller, C., P. Schedl, and T.W. Cline. 1988. Molecular characterization of daughterless, a Drosophila sex determination gene with multiple roles in development. Genes \& Dev. 2: 1666-1676.

Dambly-Chaudière, C., A. Ghysen, L.Y. Jan, and Y.N. Jan. 1988. The determination of sense organs in Drosophila: Interaction of scute with daughterless. Wilhelm Roux's Arch. Dev. Biol. 197: 419-423.

Driever, W., G. Thoma, and C. Nüsslein-Volhard. 1989. Determination of spatial domains of zygotic gene expression in the Drosophila embryo by the affinity of binding sites for the bicoid morphogen. Nature 340: 363-367.

Guan, K. and J.E. Dixon. 1991. Eukaryotic proteins expressed in Escherichia coli: An improved thrombin cleavage and purification procedure of fusion proteins with glutathione $S$ transferase. Anal. Biochem. 192: 262-267.

Ip, Y.T. and M. Levine. 1992. The role of the dorsal morphogen gradient in Drosophila embryogenesis. Sem. Dev. Biol. 3: 15-23.

Ip, Y.T., R. Kraut, M. Levine, and C.A. Rushlow. 1991. The dorsal morphogen is a sequence-specific DNA-binding pro- tein that interacts with a long-range repression element in Drosophila. Cell 64: 439-446.

Ip, Y.T., R.E. Park, D. Kosman, K. Yazdanbakhsh, and M. Levine. 1992a. dorsal-twist interactions establish snail expression in the presumptive mesoderm of the Drosophila embryo. Genes \& Dev. 6: 1518-1530.

Ip, Y.T., R.E. Park, D. Kosman, E. Bier, and M. Levine. 1992b. The dorsal gradient morphogen regulates stripes of rhomboid expression in the presumptive neuroectoderm of the Drosophila embryo. Genes \& Dev. 6: 1728-1739.

Jiang, J. and M. Levine. 1993. Binding affinities and cooperative interactions with bHLH activators delimit threshold responses to the dorsal gradient morphogen. Cell 72: 741-752.

Jiang, J., D. Kosman, Y.T. Ip, and M. Levine. 1991. The dorsal morphogen gradient regulates the mesoderm determinant twist in early Drosophila embryos. Genes \& Dev. 5: 18811891.

Knust, E., K. Tietze, and J.A. Campos-Ortega. 1987. Molecular analysis of the neurogenic locus Enhancer of split of Drosophila melanogaster. EMBO J. 6: 4113-4123.

Kosman, D., Y.T. Ip, M. Levine, and K. Arora. 1991. Establishment of the mesoderm-neuroectoderm boundary in the Drosophila embryo. Science 254: 118-122.

Lai, J.-S. and W. Herr. 1992. Ethidium bromide provides a simple tool for identifying genuine DNA-independent protein associations. Proc. Natl. Acad. Sci. 89: 6958-6962.

Leptin, M. 1991. twist and snail as positive and negative regulators during Drosophila mesoderm development. Genes \& Dev. 5: 1568-1576.

Murre, C., P.S. McCaw, and D. Baltimore. 1989. A new DNA binding and dimerization motif in immunoglobulin enhancer binding, daughterless, $M y o D$, and myc proteins. Cell 56: 777-783.

Pan, D., J.-D. Huang, and A.J. Courey. 1991. Functional analysis of the Drosophila twist promoter reveals a dorsal-binding ventral activator region. Genes \& Dev. 5: 1892-1901.

Picard, D. and W. Schaffner. 1984. A lymphocyte-specific enhancer in the mouse immunoglobulin $\kappa$ gene. Nature 307: 80-82.

Rao, Y., H. Vaessin, L.Y. Jan, and Y.-N. Jan. 1991. Neuroectoderm in Drosophila embryos is dependent on the mesoderm for positioning but not for formation. Genes \& Dev. 5: 1577 1588.

Romani, S., S. Campuzano, and J. Modolell. 1987. The achaetescute complex is expressed in neurogenic regions of Drosophila embryos. EMBO J. 6:(7) 2085-2092.

Roth, S., D. Stein, and C. Nüsslein-Volhard. 1989. A gradient of nuclear localization of the dorsal protein determines dorsoventral pattern in the Drosophila embryo. Cell 59: 11891202.

Rushlow, C.A., K. Han, J.L. Manley, and M. Levine. 1989. The graded distribution of the dorsal morphogen is initiated by selective nuclear transport in Drosophila. Cell 59: 11651177.

St Johnston, D. and C. Nüsslein-Volhard. 1992. The origin of pattern and polarity in the Drosophila embryo. Cell 68: 201219.

Simpson, P. 1983. Maternal-zygotic gene interactions during formation of the dorsoventral pattern in Drosophila embryos. Genetics 105: 615-632.

Smith, D.B. and K.S. Johnson. 1988. Single-step purification of polypeptides expressed in E. coli as fusions with glutathione S-transferase. Gene 67: 31-40.

Steward, R. 1987. Dorsal, an embryonic polarity gene in Drosophila, is homologous to the vertebrate proto-oncogene, c-rel. Science 238: 692-694. 
1989. Relocalization of the dorsal protein from the cytoplasm to the nucleus correlates with its function. Cell 59: 1179-1188.

Tautz, D. and C. Pfeifle. 1989. A nonradioactive in situ hybridization method for the localization of specific RNAs in Drosophila embryos reveals a translational control of the segmentation gene hunchback. Chromosoma 98: 81-85.

Thisse, B., C. Stoetzel, C. Gorostiza-Thisse, and F. PerrinSchmitt. 1988. Sequence of the twist gene and nuclear localization of its protein in endomesodermal cells of early Drosophila embryos. EMBO I. 7:(7) 2175-2183.

Thisse, C., F. Perrin-Schmitt, C. Stoetzel, and B. Thisse. 1991. Sequence-specific transactivation of the Drosophila twist gene by the dorsal gene product. Cell 65: 1191-1201.

Thomas, J.B., S.T. Crews, and C.S. Goodman. 1988. Molecular genetics of the single-minded locus: A gene involved in the development of the Drosophila nervous system. Cell 52: 133-141.

Van Doren, M., H.M. Ellis, and J.W. Posakony. 1991. The Drosophila extramacrochaetae protein antagonizes sequence-specific DNA binding by daughterless/achaete-scute protein complexes. Development 113: 245-255.

Villares, R. and C.V. Cabrera. 1987. The achaete-scute gene complex of $D$. melanogaster: Conserved domains in a subset of genes required for neurogenesis and their homology to myc. Cell 50: 415-424. 


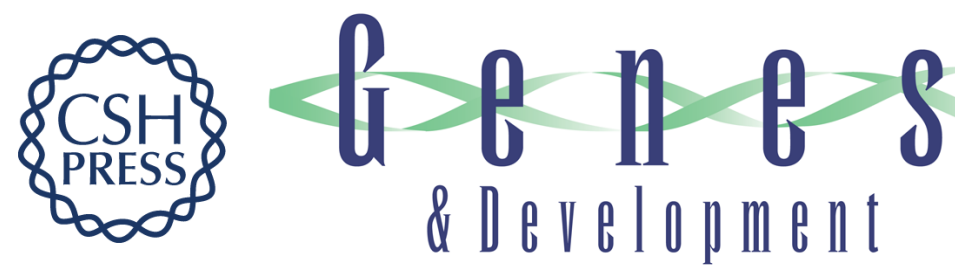

\section{Interactions between dorsal and helix-loop-helix proteins initiate the differentiation of the embryonic mesoderm and neuroectoderm in Drosophila.}

S González-Crespo and M Levine

Genes Dev. 1993, 7:

Access the most recent version at doi:10.1101/gad.7.9.1703

References This article cites 41 articles, 14 of which can be accessed free at: http://genesdev.cshlp.org/content/7/9/1703.full.html\#ref-list-1

License

Email Alerting Receive free email alerts when new articles cite this article - sign up in the box at the top Service right corner of the article or click here.

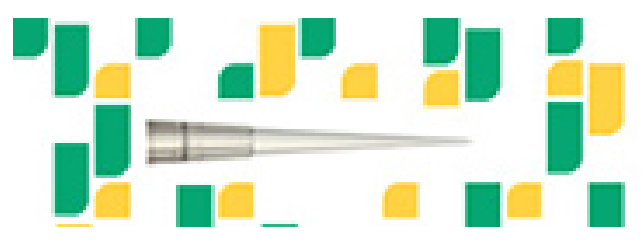

Focused on your science. 